\title{
Analysis of protease treatment-associated mutations in a group of HIV-1 subtype F infected individuals with two sequences obtained in different time points
}

Márcia Perez Resende Oliveros ${ }^{1,3^{*}}$, Clarice Gameiro da Fonseca Pachi ${ }^{2}$, Jorge Futoshi Yamamoto ${ }^{2}$, Elizabeth Cavalieri ${ }^{3}$, Maria Cecilia Araripe Sucupira ${ }^{3}$, Luis Fernandez Lopez ${ }^{1,4}$, Ricardo Sobhie Diaz ${ }^{3}$

From $16^{\text {th }}$ International Symposium on HIV and Emerging Infectious Diseases

Marseille, France. 24-26 March 2010

\section{Background}

Several studies have been performed exploring HIV-1 subtype $B$ antiretroviral resistance mutation profiles. Nonetheless, data for other HIV-1 subtypes is scarce. The aim of the present work was to analyze the subtype $\mathrm{F}$ protease antiretroviral related mutations.

\section{Methods}

Data from 38 HIV-1 subtype F individuals with sequences obtained in two different time points were analyzed. All patients presented virological failure to a protease-inhibitor containing regimen. The average of time between the first and the second sequence was 1.5 year. Most patients had changed therapy at least once between the first and the second genomic sequence. Protease subtyping was confirmed by phylogenetic analysis. $\mathrm{T}$-pared test was performed to verify possible differences in the total number of mutations and in the number of primary mutations when comparing the group containing all first sequences and the group containing all second sequences. We searched for all primary mutations present in the group of first sequences. Once a primary mutation was present in the first sequence, we verified its presence after changing therapeutic regimen.

\section{Results}

T-pared test showed that number of total mutations increased after one or more therapy changing. The number of primary mutations stayed stable, but once a primary mutation was present in the first sequence, it was commonly found also in the second sequence. That was the case of D30N, N88D, L90M and V82A.

\section{Discussion}

Our results suggest that increasing the number of total mutations after changing therapeutic schema is a trend, as well as the persistence of some primary mutations even after at least one year under a new Protease Inhibitor related selective pressure.

\section{Author details}

${ }^{1}$ School of Medicine, University of São Paulo - LIM 01 - HCFMUSP, Sao Paulo, Brazil. ${ }^{2}$ ANSP- Academic Network of São Paulo, Sao Paulo, Brazil. ${ }^{3}$ Federal University of São Paulo - Retrovirology Laboratory, Sao Paulo, Brazil. ${ }^{4}$ CIARA (Center for Internet Augmented Research and Assessment) - Florida International University, Miami, USA.

Published: 11 May 2010

doi:10.1186/1742-4690-7-S1-P50

Cite this article as: Oliveros et al:: Analysis of protease treatmentassociated mutations in a group of HIV-1 subtype F infected individuals with two sequences obtained in different time points. Retrovirology 2010 7(Suppl 1):P50.

* Correspondence: marciapr@dim.fm.usp.br

'School of Medicine, University of São Paulo - LIM 01 - HCFMUSP, Sao Paulo, Brazil 